\title{
Does routine ultrasound change management in the follow-up of patients with vesicoureteral reflux?
}

\author{
Jan K. Rudzinski, MD; ${ }^{*}$ Bryce Weber, MD, FRCSC;; Petra Wildgoose, MD;, Armando Lorenzo, MD, FRCSC,; \\ Darius Bagli, MD, FRCSC,; Walid Farhat, MD, FRCSC,; Elizabeth Harvey, MD, FRCSC,; Joao Luiz Pippi Salle, \\ $M D, F R C S C^{\S}$
}

*Faculty of Medicine, University of Calgary, Calgary, AB; ${ }^{\dagger}$ Department of Pediatric Urology, Alberta Children's Hospital, Calgary, AB; § §Department of Pediatric Urology, The Hospital for Sick Children, Calgary, AB

Cite as: Can Urol Assoc J 2013;7(7-8):e467-9. http://dx.doi.org/10.5489/cuaj.202

Published online July 2, 2013.

\section{Abstract}

Introduction: Children with vesicoureteral reflux (VUR) usually need a renal ultrasound (RUS). There is little data on the role of follow-up RUS in VUR. We evaluated the impact of follow-up RUS on the change in clinical management in patients with VUR. Methods: We prospectively analyzed children with a previous diagnosis of VUR seen in the outpatient clinic with a routine follow-up RUS within 4 months. Variables collected included: demographic data, VUR history, dysfunctional voiding symptoms and concurrent ultrasound findings. Change in management was defined as addition of new medication, nurse counselling, surgery or further investigations.

Results: The study included 114 consecutive patients. The mean patient age was 4.5 years old, mean age of VUR diagnosis was 1.7 years, with average follow-up of 2.8 years. A change in management with stable RUS occurred in 14 patients, in which the change included ordering a DMSA in 9, nurse counselling for dysfunctional voiding in 3, and booking surgery in 2 patients. Change on RUS was seen in 4 patients. Multivariable analysis showed that history of urinary tract infection (UTI) since the last follow-up visit was more significant than RUS findings.

Conclusions: The RUS findings in most patients followed for VUR remain stable or with minimal changes. The variable showing a significant effect on change in management in our study was history of UTI since the last follow-up visit rather than RUS findings. The value of follow-up RUS for children with VUR may need to be revisited.

\section{Introduction}

Vesicoureteral reflux (VUR) is defined as retrograde passage of urine from the bladder into the upper urinary tract. ${ }^{1}$ According to the American epidemiological statistics, $1 \%$ of healthy children experience VUR and $20 \%$ to $40 \%$ of children with a febrile urinary tract infection (UTI) experience VUR.-4

Complications of VUR can arise due bacterial infection of the upper urinary tract, which can increase the risk of recurrent pyelonephritis, renal scarring and subsequent renal failure. ${ }^{1}$ The goal of VUR therapy is to prevent UTIs and possible renal injury, while follow-up management is focused on identifying early signs of anatomical and functional renal abnormalities.

According to the 2010 American Urology Association

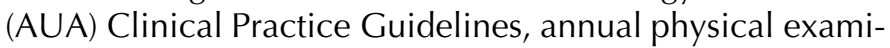
nation, including blood pressure monitoring, height and weight measurements, as well as urinalysis for proteinuria and bacteriuria, is recommended for all children with VUR until its resolution. ${ }^{5}$ Based on panel consensus reports for follow-up imaging of VUR, RUS is recommended every 12 months to monitor for renal growth and the presence of parenchymal scarring, while voiding cystourethrogram (VCUG) is recommended every 12 to 24 months. ${ }^{5}$

In patients with VUR, RUS is a cheap and non-invasive adjunct imaging modality to detect abnormal renal size, gross renal scarring, obstruction and the presence of hydronephrosis. ${ }^{2}$ However, the value of follow-up RUS for reflux-related renal scarring has not been well-established. Currently, there is no standard imaging protocol for the follow-up of patients with VUR, and practices can be quite variable. ${ }^{6}$ Since there is limited data on whether abnormal follow-up RUS findings alter management plans in children with VUR, we evaluated the impact of follow-up RUS on change in clinical management in patients with VUR.

\section{Methods}

The study was approved by the research ethics committee and patient consent was obtained via the respective parents. We evaluated 100 consecutive patients with previous diagnosis of VUR followed with routine RUS in the outpatient clinic between November 2010 and February 2011. Since 
there is evidence to show the benefit of follow-up RUS postsurgical correction of VUR to rule out ureteric obstruction, prior surgery was an exclusion criteria. ${ }^{7-9}$

The RUS was performed and interpreted at the Hospital for Sick Children in Toronto, Ontario, Canada by a pediatric radiologist. The variables collected included demographic data (age, gender and length of follow-up), VUR history, VUR grade, previous renal scars, number of previous UTIs, and UTIs since last visit, dysfunctional voiding symptoms, and concurrent ultrasound findings (i.e., renal growth, change in the grade of hydronephrosis or presence of new renal scarring).

Change in management at the time of visit was defined as the prescription of new medication, restarting antibiotic therapy, nurse counselling for voiding dysfunction, planned surgery, or further investigations, such as dimercaptosuccinic acid (DMSA) scan.

On RUS, change was defined as change in the grade of hydronephrosis, as outlined by Society of Fetal Urology Hydronephrosis Grading System, or the presence new renal scarring. ${ }^{10}$

\section{Results}

Our analysis included a total of 100 consecutive patients. The mean age was 4.5 years old and the mean age of VUR diagnosis was at 1.7 years. The mean VUR grade of the children in our study was 3 (based on initial VUR diagnosis). On average, children were followed for 2.8 years. In total, 22 patients had documented voiding dysfunction. Bilateral VUR was present in 54 patients, and renal scars were present in 27 out of 68 patients who had a previous DMSA scan performed.

Since the last follow-up visit, 16 patients had UTIs. Of these 16 patients, the mean grade of VUR was 2.8 and they had stable RUS findings; 14 (87.5\%) patients had a change in management (9 needed a DMSA scan, 3 nurse counselling for dysfunctional voiding and 2 needed surgery). Change on RUS was observed in 4 patients ( 3 with worsening grade of hydronephrosis and 1 with suspected new scars). One of these patients received change in management in the form of repeat DMSA scan to look for worsening renal scars.

When multivariable analysis for influence on change in follow-up management was conducted, only the history of UTI since the last follow up visit was found to be significant $(p<0.001)$.

\section{Discussion}

Routine follow-up RUS is commonly performed in children with VUR because of its simplicity, ability to detect large renal cortical defects, as well as renal size asymmetry. ${ }^{1}$ It is a non-invasive and non-ionizing imaging modality, which allows for quick quantitative assessment of the gross renal anatomy. However, RUS is not sensitive for detecting mild to moderate renal cortical abnormalities consistently. ${ }^{11-14}$ Currently, there is a lack of accepted standardized imaging protocols for follow-up of patients with VUR in the United States. ${ }^{6}$ In addition, physician compliance with VUR treatment and follow-up guidelines are poor. ${ }^{15,16}$ The existing variability in clinical practice guidelines indicates a lack of uniform practice recommendations. There is also paucity in evidence with regards to outcomes associated with different follow-up imaging protocols.

The AUA's recommendations regarding follow-up RUS in children with VUR are based on a panel consensus that ongoing assessment of renal growth is important in patients with VUR. ${ }^{5}$ The suggestion is based on a premise that renal growth is a marker of renal health, and the presence of gross renal scarring should be detected by follow-up ultrasonography. ${ }^{5}$ In our study, 96 out of 100 consecutive patients with established VUR had a stable follow-up RUS. Four patients had visible change on follow-up RUS and subsequently underwent change in management. Sixteen patients had stable RUS findings and the change in clinical management for this group was solely influenced by a history of UTI since the last visit. Overall, we have demonstrated that, in a small heterogenous population of children with variable VUR grades, follow-up RUS has remained stable. Screening for UTIs had a greater impact on a urologist's clinical decision-making.

In children with VUR, renal scarring and subsequent risk of reflux nephropathy are associated with UTIs, high VUR grade and abnormal lower urinary tract function. ${ }^{17}$ The odds ratio of renal scarring with acute episode of pyelonephritis in children with VUR is $2.8 .^{17}$ In a study conducted by Lowe and colleagues, the authors showed that the yield of followup RUS in children with a UTI, low-to-medium grade VUR, and normal initial RUS, has little impact on clinical management. ${ }^{18}$ Evidence to support the use of RUS in VUR is in the setting of initial workup of children with febrile UTI and VUR diagnosis alongside VCUG and DMSA. ${ }^{7-9,19}$ Additionally, RUS is beneficial as a follow-up imaging modality at discharge and 4 to 6 weeks post-surgical correction of VUR to screen for ureteral obstruction..$^{7-9}$

The approximate cost of abdominal ultrasound in Alberta is $\$ 150$ based on a cost to interpret the result by a pediatric radiologist. There are additional indirect costs, such as parental time off work, hospital parking and ultrasound technician time, which are more challenging to measure. In comparison, Charbonneau and colleagues conducted a study in the Unisted States. Authors estimated a \$7 billion annually savings by hypothetically eliminating followup RUS of uncomplicated ureteral re-implantation beyond 1 year postoperatively..$^{20}$ By potentially limiting the number of follow-up RUS on children with VUR, we could achieve significant savings for our healthcare system. 
Our study has several major limitations. The analysis was performed on only 100 patients with variable age, variable VUR grades and were not followed up over a uniform time interval. We were also only able to show one observation over a 4-month study interval. To better understand the impact of follow-up RUS on specific grades of VUR, we should conduct the study on larger sample size, and separate groups of children with uniform VUR grades followed over a longer uniform time interval.

The proponents of RUS argue that there is potential benefit of small and portable bedside RUS for screening kidneys to reduce formal ultrasound referral volume and provide quick bedside assessment of the urinary tract. ${ }^{21,22}$ However, the impact of such interventions on clinical management of children with VUR remains speculative and unclear.

\section{Conclusions}

The RUS findings in most patients followed for VUR remain stable or with minimal changes. The only major variable showing a significant effect on change in management in this prospective analysis was a history of UTI since last visit, which reflects that clinical decisions were based on recent history rather than RUS findings. In an era of restricted resources, coupled with the limitations of RUS, the value of follow-up RUS for children with VUR may need to be revisited.

Competing interests: None declared.

This paper has been peer-reviewed.

\section{References}

1. Kavoussi LR, Novick AC, Partin AW, et al. Campbell and Welsh Urology. 10th ed. Philadelphia, PA: Elsevier Saunders; 2012.

2. Thompson M, Simon SD, Sharma V, et al. Timing of Follow up Voiding Cystourethrogram in Children With Primary Vesicoureteral Reflux: Development and Application of a Clinical Algorithm. Pediatrics 2005; 115:426-34. http://dx.doi.org/10.1542/peds.2004-0927
3. Greenbaum LA, Mesrobian HGO. Vesicoureteral Reflux. Pediatr Clin N Am 2006;53:413-27. http:// dx.doi.org/10.1016/i.pcl.2006.02.010

4. Greenfield $S P, N g ~ M$, Wan J. Experience with vesicoureteral reflux in children: clinical characteristics. J Urol 1997;158:574-7. http://dx.doi.org/10.1016/S0022-5347(01)64556-7

5. Peters CA, Skoog SJ, Arant BS, et al. Summary of the AUA Guidelines on Management of Primary Vesicoureteral Reflux in Children. J Urol 2010;184:1134-44. http://dx.doi.org/10.1016/i.juro.2010.05.065

6. Gargollo $\mathrm{PC}$, Diamond DA. Therapy Insight: what nephrologists need to know about primary vesicoureteral reflux. Nat Clin Pract Nephrol 2007;3:551-63.

7. Lee MD, Lin CC, Huang FY, et al. Screening Young Children with First Febrile Urinary Tract Infection for High-grade Vesicoureteral Reflux with Renal Ultrasound Scanning and Technetium-99m-lebeled Dimercaptosuccinic Acid Scanning. J Pediatr 2009;154:797-802.

8. Giorgi UJ Jr, Bratslavsky G, Kogan BA. Febrile urinary tract infections in infants: renal ultrasonography remains necessary. J Urol 2005;173:568-70. http://dx.doi.org/10.1097/01.ju.0000149826.70405. c5

9. Jahnukainen T, Honkinen 0 , Ruuskanen 0 , et al. Ultrasonography after the first febrile urinary tract infection in children. Eur J Pediatr 2006; 165:556-9.

10. Society of Fetal Urology. SFU Grading of Infant Hydronephrosis. http://www.uab.edu/images/peduro/ SFU/sfu_grading_on_web/sfu_grading_on_web.htm. Accessed June 27, 2013.

11. Hoberman A, Charron M, Hickey RW, et al. Imaging Studies after a First Febrile Urinary Tract Infection in Young Children. N Engl J Med 2003;348:195-202.

12. Pohl HG, Belman AB. Review Article: The "Top-Down" Approach to Evaluation of Children with Febrile Urinary Tract Infection. Adv Urol 2009:783409.

13. Tasker AD, Lindsell RM, Moncrieff M. Can Ultrasound Reliably Detect Renal Scarring in Children with Urinary Tract Infection? Clin Radiol 1993;47:177-9. http://dx.doi.org/10.1016/S0009-9260(05)81157-2

14. Kangarloo H, Gold RH, Fine RN, et al. Urinary tract infection in infants and children evaluated by ultrasound. Radiology 1985; 154:367-73.

15. Cohen AL, Rivara FP, Davis R, et al. Compliance With Guidelines for the Medical Care of First Urinary Tract Infection in Infants: A Population-Based Study. Pediattics 2005;115:1474-8. http://dx.doi.org/10.1542/peds.2004-1559

16. Conway PH, Keren R. Factors associated with variability in outcomes for children hospitalized with urinary tract infection. J Pediatr 2009;154:789-96.

17. Mattoo TK. Vesicoureteral Reflux and Reflux Nephropathy. Adv Chronic Kidney Dis 2011;18:348-54.

18. Lowe LH, Patel MN, Gatti JM, et al. Utility of Follow-up Renal Sonography in Children With Vesicoureteral Reflux and Normal Initial Sonogram. Pediatrics 2004;113:548-50. http://dx.doi.org/10.1542/ peds. 113.3 .548

19. Merguerian PA, Jamal MA, Agarwal SK, et al. Utility of SPECT DMSA renal scanning in the evaluation of children with primary vesicoureteral reflux. Pediatr Urol 1999;53:1024-8.

20. Charbonneau SG, Tackett LD, Gray EH, et al. Is Long-Term Sonograhic Follow Up Necessary After Uncomplicated Ureteral Reimplantation in Children? J Urol 2005;174:1429-32. http://dx.doi. org/10.1097/01.ju.0000173128.73742.bc

21. Goldberg E, Gilbert BR. Office Ultrasound for the Urologist. Curr Urol Rep 2012;13:460-6. http://dx.doi.org/10.1007/s11934-012-0280-y

22. Moslemi MK, Mahfoozi B. Urologist-operated ultrasound and its use in urological outpatient clinics. Patients Prefer Adherence 2011;5:85-8.

Correspondence: Dr. Bryce Weber , Alberta Children's Hospital, 2888 Shagannapi Trail NW Division of Pediatric Urology Calgary, AB T3B 6A8; bryce.weber@albertahealthservices.ca 